\title{
Numerical Solutions of Nonlinear Fractional Partial Differential Equations Arising in Spatial Diffusion of Biological Populations
}

\author{
Jagdev Singh, ${ }^{1}$ Devendra Kumar, ${ }^{2}$ and Adem Kılıçman ${ }^{3}$ \\ ${ }^{1}$ Department of Mathematics, Jagannath University, Jaipur-303901, Rajasthan, India \\ ${ }^{2}$ Department of Mathematics, Jagannath Gupta Institute of Engineering and Technology, Jaipur-302022, Rajasthan, India \\ ${ }^{3}$ Department of Mathematics and Institute for Mathematical Research University Putra Malaysia, 43400 Serdang, \\ Selangor, Malaysia
}

Correspondence should be addressed to Adem Kılıçman; akilic@upm.edu.my

Received 16 February 2014; Accepted 8 July 2014; Published 5 August 2014

Academic Editor: Hassan Eltayeb

Copyright (C) 2014 Jagdev Singh et al. This is an open access article distributed under the Creative Commons Attribution License, which permits unrestricted use, distribution, and reproduction in any medium, provided the original work is properly cited.

\begin{abstract}
The main aim of this work is to present a user friendly numerical algorithm based on homotopy perturbation Sumudu transform method for nonlinear fractional partial differential arising in spatial diffusion of biological populations in animals. The movements are made generally either by mature animals driven out by invaders or by young animals just reaching maturity moving out of their parental territory to establish breeding territory of their own. The homotopy perturbation Sumudu transform method is a combined form of the Sumudu transform method and homotopy perturbation method. The obtained results are compared with Sumudu decomposition method. The numerical solutions obtained by the proposed method indicate that the approach is easy to implement and accurate. These results reveal that the proposed method is computationally very attractive.
\end{abstract}

\section{Introduction}

Nonlinear phenomena have important effects on applied mathematics, biology, and physics and are related to engineering; many such physical phenomena are modeled in terms of nonlinear partial differential equations. For example, the degenerate parabolic nonlinear partial differential equations arising in the spatial diffusion of biological populations are given as

$$
U_{t}=G(U)_{x x}+G(U)_{y y}+f(t, x, y, U), \quad t \geq 0, x, y \in R
$$

with initial condition $U(x, y, 0)=U_{0}(x, y)$, where $U$ denotes the population density and $f$ represents the population supply due to birth and death. It is worth mentioning that (1) with $f(t, x, y, U)=-k U^{q}(x, y, t)$, where $k \geq 0,0<$ $q<1$, arises in the study of flow through porous media $[1,2]$. Moreover, the problem (1) leads to Malthusian law [3] for $f(t, x, y, U)=c_{1} U(x, y, t)$ and Verhulst law [3] for $f(t, x, y, U)=c_{2} U(x, y, t)-c_{3} U^{2}(x, y, t)$, where $c_{1}, c_{2}, c_{3}$ are positive constants. We consider the model as an example in the population of animals. The movements are made generally either by mature animals driven out by invaders or by young animals just reaching maturity moving out of their parental territory to establish breeding territory of their own. In both cases, it is much more plausible to suppose that they will be directed towards nearby vacant territory. Therefore, movement will take place almost exclusively "down" the population density gradient and will be much more rapid at high population densities than at low ones. In an attempt to model this situation, they considered a walk through a rectangular grid, in which at each step an animal may either stay at its present location or may move in the direction of lowest population density. We consider the more general form of time fractional biological equation by taking $f(U)=$ $h U^{g}\left(1-r U^{h}\right)$ as

$$
\begin{array}{r}
D_{t}^{\alpha} U=D_{x}^{2} U^{2}+D_{y}^{2} U^{2}+h U^{g}\left(1-r U^{h}\right), \\
0<\alpha \leq 1, \quad t \geq 0, \quad x, y \in R,
\end{array}
$$


with initial condition $U(x, y, 0)=U_{0}(x, y)$, where $U$ denotes the populations density and $f$ represents the population supply due to births and deaths. The most important advantage of using fractional differential equations in mathematical modelling is their nonlocal property. It is well known that the integer order differential operator is a local operator, but the fractional order differential operator is nonlocal. This means that the next state of a system depends not only upon its current state but also upon all of its historical states. This is more realistic and it is one reason why fractional calculus has become more and more popular [4-10]. There are several methods to solve the fractional biological equations, such as Adomian's decomposition method (ADM) [11] and homotopy analysis method (HAM) [12]. The optimal homotopy analysis method (OHAM) has been applied for solving nonlinear problems [13-15]. The homotopy perturbation method (HPM) has first proposed by He [16-18] for solving various linear and nonlinear initial and boundary value problems. The HPM was also investigated by many researchers to handle nonlinear equations arising in science and engineering [1922]. The Laplace transform and Sumudu transform are totally incapable of handling nonlinear equations because of the difficulties that are caused by the nonlinear terms. Various ways have been proposed recently to deal with such nonlinearities such as the Laplace decomposition algorithm $[23,24]$, the homotopy perturbation transform method (HPTM) [25], Sumudu decomposition method (SDM) [26], and the homotopy perturbation Sumudu transform method (HPSTM) [27] to produce highly effective techniques for solving many nonlinear problems.

In this paper, we implement the homotopy perturbation Sumudu transform method (HPSTM) to find the analytical and numerical solutions of the fractional biological population models. The HPSTM is an elegant combination of the Sumudu transform method, the HPM and Adomian's polynomials. Moreover, we apply the Sumudu decomposition method (SDM) to the same problems to compare the results. The SDM is a combined form of Sumudu transform method and Adomian's decomposition method (ADM) which was first introduced by Adomian $[28,29]$. Adomian's polynomials are studied by Jafari et al. [30, 31] for handling nonlinear equations and showed that the Adomian's polynomials and He's polynomials are same. The numerical results are depicted through the graphical illustrations. The proposed techniques provide the solution in a rapid convergent series which may lead to the solution in a closed form. The advantage of these methods is their capability of combining two powerful methods for obtaining exact and approximate solutions for nonlinear equations.

\section{Basic Definitions of Fractional Calculus}

In this section, we mention the following basic definitions of fractional calculus and Sumudu transform.

Definition 1. The Riemann-Liouville fractional integral operator of order $\alpha>0$, of a function $f(t) \in C_{\mu}, \mu \geq-1$, is defined as [4]

$$
\begin{aligned}
J^{\alpha} f(t) & =\frac{1}{\Gamma(\alpha)} \int_{0}^{t}(t-\tau)^{\alpha-1} f(\tau) d \tau, \quad(\alpha>0), \\
J^{0} f(t) & =f(t) .
\end{aligned}
$$

For the Riemann-Liouville fractional integral we have

$$
J^{\alpha} t^{\gamma}=\frac{\Gamma(\gamma+1)}{\Gamma(\gamma+\alpha+1)} t^{\alpha+\gamma} .
$$

Definition 2. The fractional derivative of $f(t)$ in the Caputo sense is defined as [5]

$$
\begin{aligned}
D_{t}^{\alpha} f(t) & =J^{m-\alpha} D^{n} f(t) \\
& =\frac{1}{\Gamma(n-\alpha)} \int_{0}^{t}(t-\tau)^{m-\alpha-1} f^{(m)}(\tau) d \tau,
\end{aligned}
$$

for $m-1<\alpha \leq m, m \in N$, and $t>0$.

Definition 3. The Sumudu transform [32] is defined over the set of functions:

$$
\begin{gathered}
A=\left\{f(t)\left|\exists M, \tau_{1}, \tau_{2}>0,\right| f(t) \mid<M e^{|t| / \tau_{j}},\right. \\
\text { if } \left.t \in(-1)^{j} \times[0, \infty)\right\},
\end{gathered}
$$

by the following formula:

$$
\bar{f}(u)=S[f(t)]=\int_{0}^{\infty} f(u t) e^{-t} d t, \quad u \in\left(-\tau_{1}, \tau_{2}\right) .
$$

Some of the important properties of the Sumudu transform were established by Asiru [33]. Further, fundamental properties of this transform were established by Belgacem et al. [34] and Belgacem and Karaballi [35]. In fact it was shown that there is strong relationship between Sumudu and other integral transform; see Kılıçman et al. [36]. In particular the relationship between Sumudu transform and Laplace transforms was presented in K1lıcman and Gadain [37]. The Sumudu transform has scale and unit preserving properties, so it can be used to solve problems without resorting to a new frequency domain.

Definition 4. The Sumudu transform of the Caputo fractional derivative is defined as follows [38]:

$$
\begin{array}{r}
S\left[D_{t}^{\alpha} f(t)\right]=u^{-\alpha} S[f(t)]-\sum_{k=0}^{m} u^{-\alpha+k} f^{(k)}(0+), \\
(m-1<\alpha \leq m) .
\end{array}
$$

\section{Homotopy Perturbation Sumudu Transform Method (HPSTM)}

To illustrate the basic idea of this method, we consider a general fractional nonlinear nonhomogeneous partial differential equation with the initial condition of the form:

$$
\begin{gathered}
D_{t}^{\alpha} U(x, t)+R U(x, t)+N U(x, t)=g(x, t), \\
U(x, 0)=f(x),
\end{gathered}
$$


where $D_{t}^{\alpha} U(x, t)$ is the Caputo fractional derivative of the function $U(x, t), R$ is the linear differential operator, $N$ represents the general nonlinear differential operator, and $g(x, t)$, is the source term.

Applying the Sumudu transform (denoted in this paper by $S$ ) on both sides of (9), we get

$$
S\left[D_{t}^{\alpha} U(x, t)\right]+S[R U(x, t)]+S[N U(x, t)]=S[g(x, t)] .
$$

Using the differentiation property of the Sumudu transform, we have

$$
\begin{aligned}
S[U(x, t)]= & f(x)+u^{\alpha} S[g(x, t)] \\
& -u^{\alpha} S[R U(x, t)+N U(x, t)] .
\end{aligned}
$$

Operating with the Sumudu inverse on both sides of (12) gives

$$
U(x, t)=G(x, t)-S^{-1}\left[u^{\alpha} S[R U(x, t)+N U(x, t)]\right],
$$

where $G(x, t)$ represents the term arising from the source term and the prescribed initial conditions. Now we apply the HPM

$$
U(x, t)=\sum_{n=0}^{\infty} p^{n} U_{n}(x, t),
$$

and the nonlinear term can be decomposed as

$$
N U(x, t)=\sum_{n=0}^{\infty} p^{n} A_{n}
$$

for some Adomian's polynomials $A_{n}$ that are given by

$$
A_{n}=\frac{1}{n !} \frac{d^{n}}{d p^{n}}\left[N\left(\sum_{i=0}^{\infty} p^{i} U_{i}\right)\right]_{p=0}, \quad n=0,1,2, \ldots
$$

Substituting (14) and (15) in (13), we get

$$
\begin{array}{r}
\sum_{n=0}^{\infty} p^{n} U_{n}(x, t)=G(x, t)-p\left(S ^ { - 1 } \left[u ^ { \alpha } S \left[R \sum_{n=0}^{\infty} p^{n} U_{n}(x, t)\right.\right.\right. \\
\left.\left.\left.+\sum_{n=0}^{\infty} p^{n} A_{n}\right]\right]\right),
\end{array}
$$

which is the coupling of the Sumudu transform method, the HPM, and Adomian's polynomials. Comparing the coefficients of like powers of $p$, the following approximations are obtained:

$$
\begin{aligned}
& p^{0}: U_{0}(x, t)=G(x, t), \\
& p^{1}: U_{1}(x, t)=-S^{-1}\left[u^{\alpha} S\left[R U_{0}(x, t)+A_{0}\right]\right], \\
& p^{2}: U_{2}(x, t)=-S^{-1}\left[u^{\alpha} S\left[R U_{1}(x, t)+A_{1}\right]\right], \\
& p^{3}: U_{3}(x, t)=-S^{-1}\left[u^{\alpha} S\left[R U_{2}(x, t)+A_{2}\right]\right],
\end{aligned}
$$

Proceeding in the same manner, the rest of the components $U_{n}(x, t)$ can be completely found and the series solution is thus entirely determined. Finally, we approximate the analytical solution $U(x, t)$ by truncated series as

$$
U(x, t)=\lim _{N \rightarrow \infty} \sum_{n=0}^{N} U_{n}(x, t) .
$$

\section{Sumudu Decomposition Method (SDM)}

We consider a general fractional nonlinear nonhomogeneous partial differential equation with the initial condition of the form:

$$
\begin{gathered}
D_{t}^{\alpha} U(x, t)+R U(x, t)+N U(x, t)=g(x, t), \\
U(x, 0)=f(x),
\end{gathered}
$$

where $D_{t}^{\alpha} U(x, t)$ is the Caputo fractional derivative of the function $U(x, t), R$ is the linear differential operator, $N$ represents the general nonlinear differential operator, and $g(x, t)$ is the source term.

Taking the Sumudu transform on both sides of (20), we get

$$
S\left[D_{t}^{\alpha} U(x, t)\right]+S[R U(x, t)]+S[N U(x, t)]=S[g(x, t)] .
$$

Using the differentiation property of the Sumudu transform and the above initial conditions, we have

$$
\begin{aligned}
S[U(x, t)]= & f(x)+u^{\alpha} S[g(x, t)] \\
& -u^{\alpha} S[R U(x, t)+N U(x, t)] .
\end{aligned}
$$

Now, applying the inverse Sumudu transform on both sides of (23), we get

$$
U(x, t)=G(x, t)-S^{-1}\left[u^{\alpha} S[R U(x, t)+N U(x, t)]\right],
$$

where $G(x, t)$ represents the term arising from the source term and the prescribed initial conditions.

The second step in SDM is that we represent solution as an infinite series given below:

$$
U(x, t)=\sum_{n=0}^{\infty} U_{n}(x, t)
$$

and the nonlinear term can be decomposed as

$$
N U(x, t)=\sum_{n=0}^{\infty} A_{n}
$$

where $A_{n}$ are Adomian polynomials of $U_{0}, U_{1}, U_{2}, \ldots, U_{n}$ and it can be calculated by formula given below:

$$
A_{n}=\frac{1}{n !} \frac{d^{n}}{d \lambda^{n}}\left[N\left(\sum_{i=0}^{\infty} \lambda^{i} U_{i}\right)\right]_{\lambda=0}, \quad n=0,1,2, \ldots
$$


Using (25) and (26) in (24), we get

$$
\begin{aligned}
\sum_{n=0}^{\infty} U_{n}(x, t)= & G(x, t) \\
& -S^{-1}\left[u^{\alpha} S\left[R \sum_{n=0}^{\infty} U_{n}(x, t)+\sum_{n=0}^{\infty} A_{n}\right]\right] .
\end{aligned}
$$

On comparing both sides of (28), we get

$$
\begin{aligned}
& U_{0}(x, t)=G(x, t), \\
& U_{1}(x, t)=-S^{-1}\left[u^{\alpha} S\left[R U_{0}(x, t)+A_{0}\right]\right], \\
& U_{2}(x, t)=-S^{-1}\left[u^{\alpha} S\left[R U_{1}(x, t)+A_{1}\right]\right] .
\end{aligned}
$$

In general the recursive relation is given by

$$
U_{n+1}(x, t)=-S^{-1}\left[u^{\alpha} S\left[R U_{n}(x, t)+A_{n}\right]\right], \quad n \geq 0 .
$$

Now first of all applying the Sumudu transform of the right hand side of (30) then applying the inverse Sumudu transform, we get the values of $U_{0}, U_{1}, U_{2}, \ldots, U_{n}$, respectively.

\section{Numerical Examples}

In this section, we demonstrate the performance and efficiency of HPSTM by giving three examples of time-fractional biological models and compare the results with the results obtained with help of SDM.

Example 1. We consider the following time-fractional biological population model:

$$
D_{t}^{\alpha} U(x, y, t)=D_{x}^{2} U^{2}+D_{y}^{2} U^{2}+h U, \quad 0<\alpha \leq 1,
$$

subject to the initial condition

$$
U(x, y, 0)=\sqrt{x y} .
$$

Operating with the Sumudu transform on both sides of (31), we have

$$
S[U(x, y, t)]=\sqrt{x y}+u^{\alpha} S\left[D_{x}^{2} U^{2}+D_{y}^{2} U^{2}+h U\right] .
$$

The inverse of the Sumudu transform gives that

$$
U(x, y, t)=\sqrt{x y}+S^{-1}\left[u^{\alpha} S\left[D_{x}^{2} U^{2}+D_{y}^{2} U^{2}+h U\right]\right] .
$$

According to the HPM, we construct the following homotopy:

$$
\begin{aligned}
\sum_{n=0}^{\infty} p^{n} U_{n}(x, y, t)= & \sqrt{x y}+p S^{-1} \\
& \times\left(u ^ { \alpha } S \left[\sum_{n=0}^{\infty} p^{n} A_{n}\right.\right. \\
& \left.\left.+h \sum_{n=0}^{\infty} p^{n} U_{n}(x, y, t)\right]\right),
\end{aligned}
$$

where $A_{n}$ are Adomian's polynomials that represent the nonlinear terms. The first few components of Adomian's polynomials are given by

$$
\begin{aligned}
& A_{0}=D_{x}^{2} U_{0}^{2}+D_{y}^{2} U_{0}^{2}, \\
& A_{1}=2 D_{x}^{2}\left(U_{0} U_{1}\right)+2 D_{y}^{2}\left(U_{0} U_{1}\right), \ldots
\end{aligned}
$$

Comparing the coefficient of like power of $p$, we have

$$
\begin{aligned}
& p^{0}: U_{0}(x, y, t)=\sqrt{x y}, \\
& p^{1}: U_{1}(x, y, t)=S^{-1}\left[u^{\alpha} S\left[A_{0}+h U_{0}\right]\right]=\sqrt{x y} \frac{h t^{\alpha}}{\Gamma(\alpha+1)}, \\
& p^{2}: U_{2}(x, y, t)=S^{-1}\left[u^{\alpha} S\left[A_{1}+h U_{1}\right]\right]=\sqrt{x y} \frac{\left(h t^{\alpha}\right)^{2}}{\Gamma(2 \alpha+1)}, \\
& p^{3}: U_{3}(x, y, t)=S^{-1}\left[u^{\alpha} S\left[A_{2}+h U_{2}\right]\right]=\sqrt{x y} \frac{\left(h t^{\alpha}\right)^{3}}{\Gamma(3 \alpha+1)},
\end{aligned}
$$

and so on.

Making use of (37), the series solution $U(x, y, t)$ is given as

$$
\begin{aligned}
& U(x, y, t)= \lim _{p \rightarrow 1} \sum_{n=0}^{\infty} p^{n} U_{n}(x, y, t) \\
&=\sqrt{x y}\left[1+\frac{h t^{\alpha}}{\Gamma(\alpha+1)}+\frac{h^{2} t^{2 \alpha}}{\Gamma(2 \alpha+1)}\right. \\
&\left.+\frac{h^{3} t^{3 \alpha}}{\Gamma(3 \alpha+1)}+\cdots\right] .
\end{aligned}
$$

Setting $\alpha=1$ in (38), we reproduce the solution of the problem as follows:

$$
U(x, y, t)=\sqrt{x y}\left[1+\frac{h t}{1 !}+\frac{(h t)^{2}}{2 !}+\frac{(h t)^{3}}{3 !}+\cdots\right],
$$

which converge very rapidly to the exact solution $U(x, y, t)=$ $\sqrt{x y} e^{h t}$.

According to SDM and to (25) and (34), the iteration formula for (31) is given by

$$
\begin{aligned}
\sum_{n=0}^{\infty} U_{n}(x, y, t)= & \sqrt{x y}+S^{-1} \\
& \times\left(u^{\alpha} S\left[\sum_{n=0}^{\infty} A_{n}+h \sum_{n=0}^{\infty} U_{n}(x, y, t)\right]\right),
\end{aligned}
$$

where $A_{n}$ are Adomian's polynomials that represent the nonlinear terms. The first few components of Adomian's polynomials are given by

$$
\begin{aligned}
& A_{0}=D_{x}^{2} U_{0}^{2}+D_{y}^{2} U_{0}^{2}, \\
& A_{1}=2 D_{x}^{2}\left(U_{0} U_{1}\right)+2 D_{y}^{2}\left(U_{0} U_{1}\right), \ldots
\end{aligned}
$$


The recursive relation is given below:

$$
\begin{aligned}
U_{0}(x, y, t) & =\sqrt{x y}, \\
U_{1}(x, y, t) & =S^{-1}\left[u^{\alpha} S\left[A_{0}+h U_{0}\right]\right], \\
U_{n+1}(x, y, t) & =S^{-1}\left[u^{\alpha} S\left[A_{n}+h U_{n}\right]\right] .
\end{aligned}
$$

The other components of the solution can be easily found by using above recursive relation:

$$
\begin{aligned}
& U_{1}(x, y, t)=\sqrt{x y} \frac{h t^{\alpha}}{\Gamma(\alpha+1)}, \\
& U_{2}(x, y, t)=\sqrt{x y} \frac{\left(h t^{\alpha}\right)^{2}}{\Gamma(2 \alpha+1)}, \\
& U_{3}(x, y, t)=\sqrt{x y} \frac{\left(h t^{\alpha}\right)^{3}}{\Gamma(3 \alpha+1)},
\end{aligned}
$$

and so on.

Therefore, the decomposition series solutions is given by

$$
\begin{gathered}
U(x, y, t)=\sqrt{x y}\left[1+\frac{h t^{\alpha}}{\Gamma(\alpha+1)}+\frac{h^{2} t^{2 \alpha}}{\Gamma(2 \alpha+1)}\right. \\
\left.+\frac{h^{3} t^{3 \alpha}}{\Gamma(3 \alpha+1)}+\cdots\right],
\end{gathered}
$$

which is the same solution as obtained by using HPSTM and setting $\alpha=1$, it converge to the exact solution $U(x, y, t)=$ $\sqrt{x y} e^{h t}$.

The numerical results for the time-fractional biological population model (31) obtained by using the HPSTM, SDM, and the exact solution for various values of $t, x$, and $\alpha$ with $y=1$ and $h=1$ are shown by Figures $1(\mathrm{a})-1(\mathrm{~d})$ and those for various values of $t$ at $x=1, y=1, h=1$, and $\alpha=1$ are depicted in Figure 2 and those for different values of $t$ and $\alpha$ at $x=1, y=1$, and $h=1$ are shown in Figure 3. It is observed from Figure 1 that $U(x, y, t)$ increases with the increase in both $x$ and $t$ for $\alpha=0.5,0.75$, and 1 with $y=1$ and $h=1$. It is seen from Figure 3 that as the value of $\alpha$ decreases, the numerical value of $U(x, y, t)$ increases. It can also be seen from Figure 1 that the solution obtained by using the present method is nearly identical with the exact solution. From Figure 2, we can easily observe that the results obtained with help of HPSTM and SDM are in a full agreement with the exact solution. It is to be noted that only the seventh order term of the HPSTM and SDM was used in evaluating the approximate solutions for Figures 1 and 2. It is evident that the efficiency of the present method can be dramatically enhanced by computing further terms of $U(x, y, t)$ when the HPSTM and SDM are used.

Example 2. Next, we consider the time-fractional biological population model in the following form:

$$
D_{t}^{\alpha} U(x, y, t)=D_{x}^{2} U^{2}+D_{y}^{2} U^{2}+U, \quad 0<\alpha \leq 1,
$$

subject to initial condition

$$
U(x, y, 0)=\sqrt{\sin x \sinh y} .
$$

By applying the HPSTM, we have

$$
\begin{aligned}
\sum_{n=0}^{\infty} p^{n} U_{n}(x, y, t)= & \sqrt{\sin x \sinh y} \\
& +p S^{-1}\left(u ^ { \alpha } S \left[\sum_{n=0}^{\infty} p^{n} A_{n}\right.\right. \\
& \left.\left.+\sum_{n=0}^{\infty} p^{n} U_{n}(x, y, t)\right]\right) .
\end{aligned}
$$

Equating the like powers of $p,(47)$ yields

$$
\begin{aligned}
& p^{0}: U_{0}(x, y, t)=\sqrt{\sin x \sinh y}, \\
& p^{1}: U_{1}(x, y, t)=\sqrt{\sin x \sinh y} \frac{t^{\alpha}}{\Gamma(\alpha+1)}, \\
& p^{2}: U_{2}(x, y, t)=\sqrt{\sin x \sinh y} \frac{t^{2 \alpha}}{\Gamma(2 \alpha+1)}, \\
& p^{3}: U_{3}(x, y, t)=\sqrt{\sin x \sinh y} \frac{t^{3 \alpha}}{\Gamma(3 \alpha+1)},
\end{aligned}
$$

and so on.

Taking use of (48), the series solution $U(x, y, t)$ is presented as

$$
\begin{aligned}
U(x, y, t)= & \lim _{p \rightarrow 1} \sum_{n=0}^{\infty} p^{n} U_{n}(x, y, t) \\
=\sqrt{\sin x \sinh y} & {\left[1+\frac{t^{\alpha}}{\Gamma(\alpha+1)}+\frac{t^{2 \alpha}}{\Gamma(2 \alpha+1)}\right.} \\
& \left.+\frac{t^{3 \alpha}}{\Gamma(3 \alpha+1)}+\cdots\right] .
\end{aligned}
$$




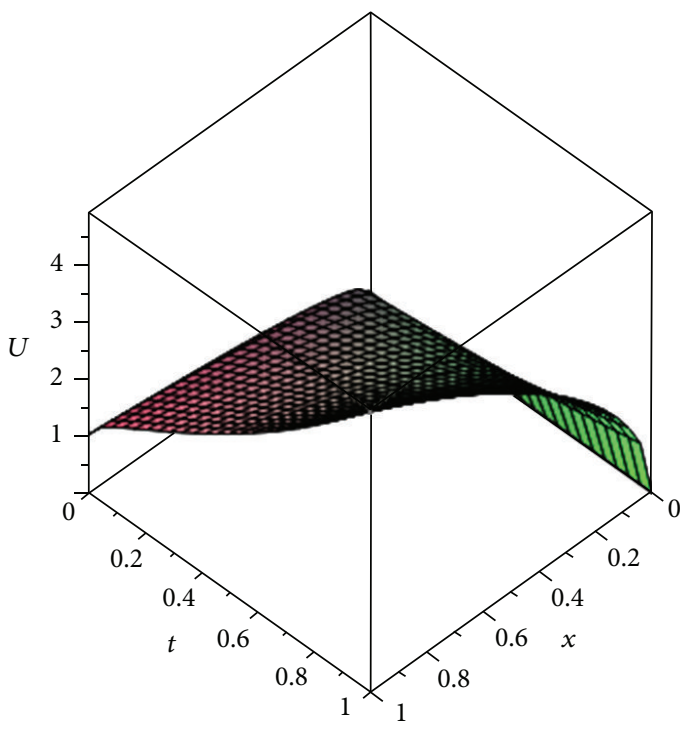

(a)

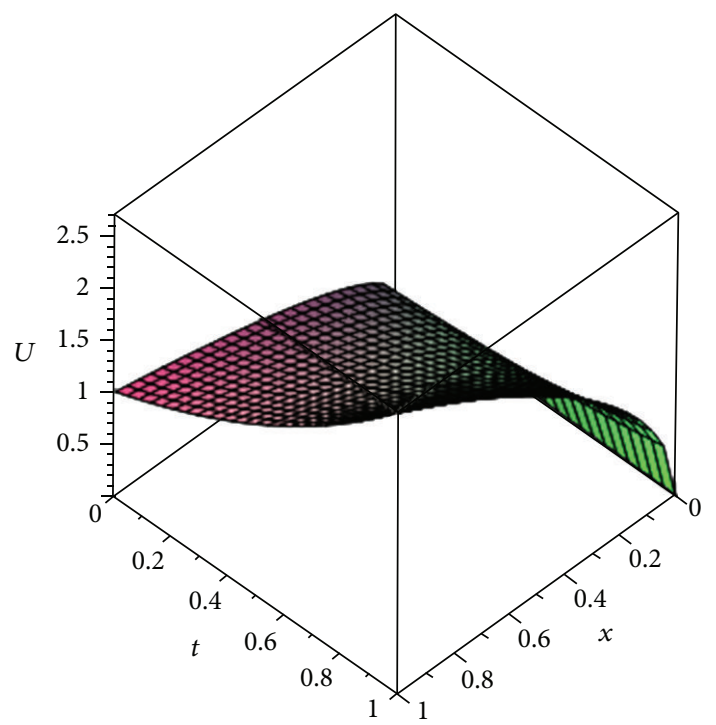

(c)

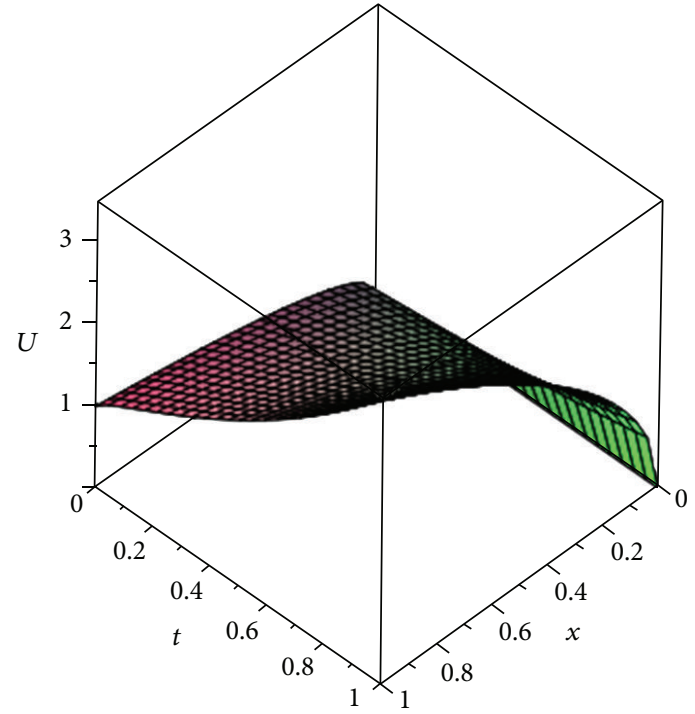

(b)



(d)

Figure 1: The behavior of the $U(x, y, t)$ with respect to $x$ and $t$ is obtained with $y=1$ and $h=1$, when (a) $\alpha=0.5$; (b) $\alpha=0.75$; (c) $\alpha=1$; (d) exact solution.

Putting $\alpha=1$ in (49), we reproduce the solution of the problem as follows:

$$
u(x, y, t)=\sqrt{\sin x \sinh y}\left[1+\frac{t}{1 !}+\frac{t^{2}}{2 !}+\frac{t^{3}}{3 !}+\cdots\right],
$$

which converge very rapidly to the exact solution $U(x, y, t)=$ $\sqrt{\sin x \sinh y} e^{t}$.

According to SDM, we have

$$
\begin{aligned}
\sum_{n=0}^{\infty} U_{n}(x, y, t)= & \sqrt{\sin x \sinh y} \\
& +S^{-1}\left(u^{\alpha} S\left[\sum_{n=0}^{\infty} A_{n}+\sum_{n=0}^{\infty} U_{n}(x, y, t)\right]\right) .
\end{aligned}
$$

The recursive relation is given below:

$$
\begin{aligned}
U_{0}(x, y, t) & =\sqrt{\sin x \sinh y}, \\
U_{1}(x, y, t) & =S^{-1}\left[u^{\alpha} S\left[A_{0}+U_{0}\right]\right], \\
U_{n+1}(x, y, t) & =S^{-1}\left[u^{\alpha} S\left[A_{n}+U_{n}\right]\right] .
\end{aligned}
$$

The other components of the solution can be easily found by using above recursive relation:

$$
\begin{aligned}
& U_{0}(x, y, t)=\sqrt{\sin x \sinh y}, \\
& U_{1}(x, y, t)=\sqrt{\sin x \sinh y} \frac{t^{\alpha}}{\Gamma(\alpha+1)},
\end{aligned}
$$




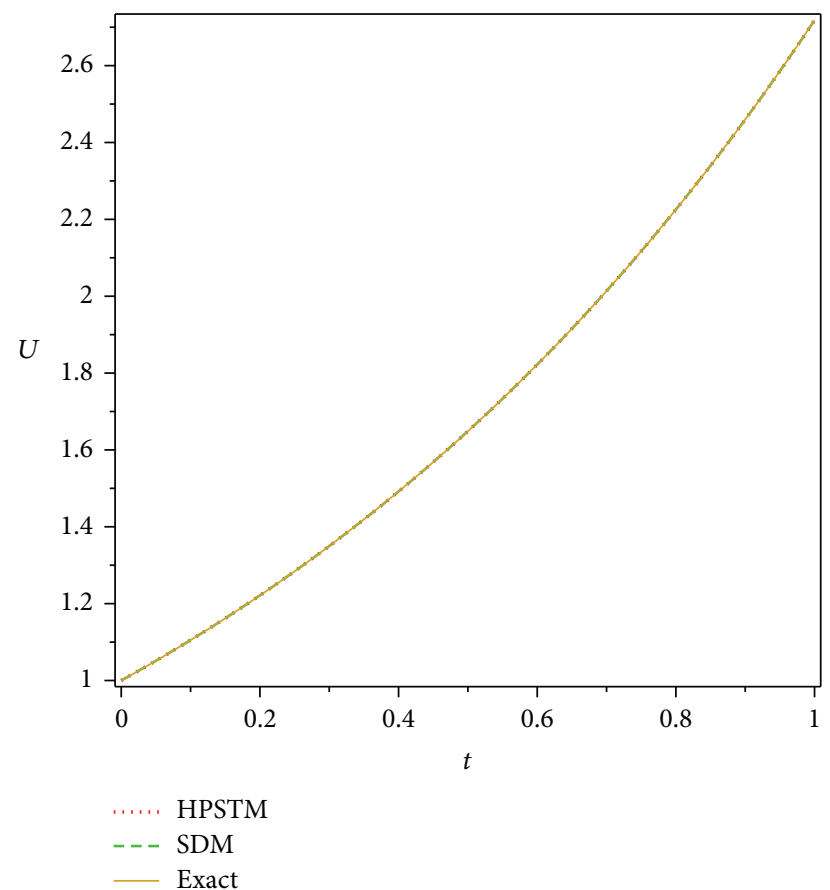

Figure 2: Plots of $U(x, y, t)$ versus $t$ at $h=1, x=1, y=1$, and $\alpha=1$.

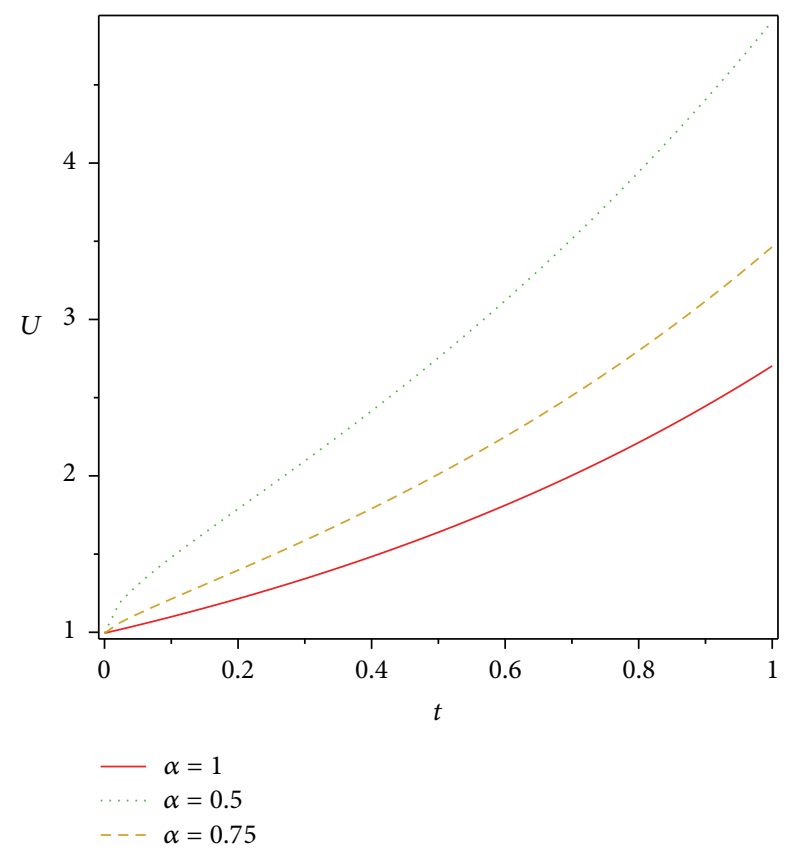

Figure 3: Plots of $U(x, y, t)$ versus $t$ at $h=1, x=1$, and $y=1$ for different values of $\alpha$.

$$
\begin{aligned}
& U_{2}(x, y, t)=\sqrt{\sin x \sinh y} \frac{t^{2 \alpha}}{\Gamma(2 \alpha+1)}, \\
& U_{3}(x, y, t)=\sqrt{\sin x \sinh y} \frac{t^{3 \alpha}}{\Gamma(3 \alpha+1)},
\end{aligned}
$$

Thus, the decomposition series solutions is given by

$$
\begin{aligned}
U(x, y, t)=\sqrt{\sin x \sinh y}\left[1+\frac{t^{\alpha}}{\Gamma(\alpha+1)}+\frac{t^{2 \alpha}}{\Gamma(2 \alpha+1)}\right. \\
\left.+\frac{t^{3 \alpha}}{\Gamma(3 \alpha+1)}+\cdots\right]
\end{aligned}
$$

which is the same solution as obtained by employing HPSTM and setting $\alpha=1$; it converses to the exact solution $U(x, y, t)=\sqrt{\sin x \sinh y} e^{t}$.

The numerical results for the time-fractional biological population model (45) obtained with the help of HPSTM, SDM, and the exact solution for various values of $t, x$, and $\alpha$ with $y=1$ are described through Figures 4(a)-4(d) and those for various values of $t$ at $x=1, y=1$, and $\alpha=1$ are given in Figure 5 and those for different values of $t$ and $\alpha$ at $x=1$ and $y=1$ are depicted in Figure 6. It is observed from Figure 4 that $U(x, y, t)$ increases with the increase in both $x$ and $t$ for $\alpha=0.5,0.75$, and 1 with $y=1$. It is to be observed from Figure 6 that as the value of $\alpha$ decrease, the value of $U(x, y, t)$ increases. It can also be seen from Figure 4 that the solution obtained by using the present method is nearly identical with the exact solution. From Figure 5, we can easily observe that the results obtained with help of HPSTM and SDM are in a full agreement with the exact solution. It is to be noted that only the seventh order term of the HPSTM and SDM was used in evaluating the approximate solutions for Figures 4 and 5.

Example 3. Finally, we consider the following time-fractional biological population model:

$$
\begin{array}{r}
D_{t}^{\alpha} U=D_{x}^{2} U^{2}+D_{y}^{2} U^{2}-U(x, y, t)\left(1+\frac{8}{9} U(x, y, t)\right), \\
0<\alpha \leq 1,
\end{array}
$$

subject to the initial condition

$$
U(x, y, 0)=e^{(x+y) / 3} .
$$

By applying the HPSTM, we have

$$
\begin{aligned}
\sum_{n=0}^{\infty} p^{n} U_{n}(x, y, t)= & e^{(x+y) / 3} \\
& +p S^{-1}\left(u ^ { \alpha } S \left[\sum_{n=0}^{\infty} p^{n} A_{n}\right.\right. \\
& \left.\left.\quad-\sum_{n=0}^{\infty} p^{n} U_{n}(x, y, t)\right]\right) .
\end{aligned}
$$

and so on. 


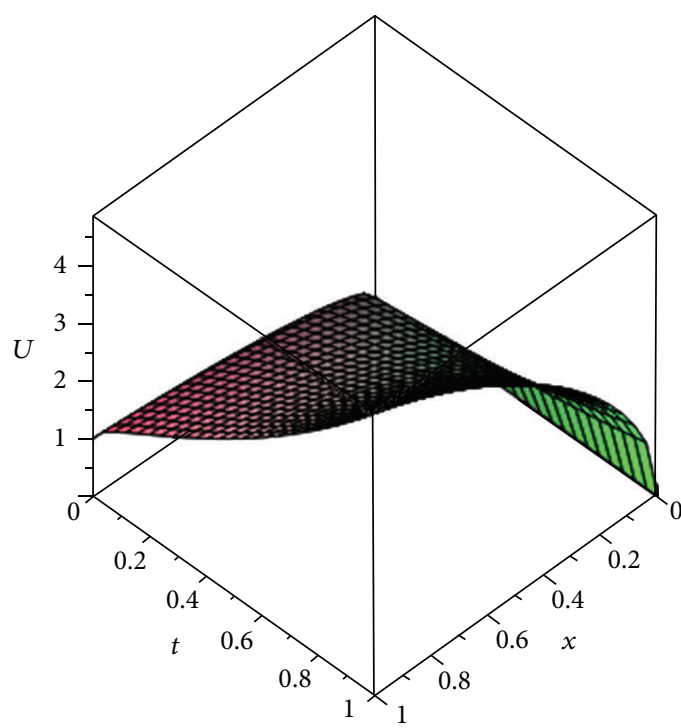

(a)

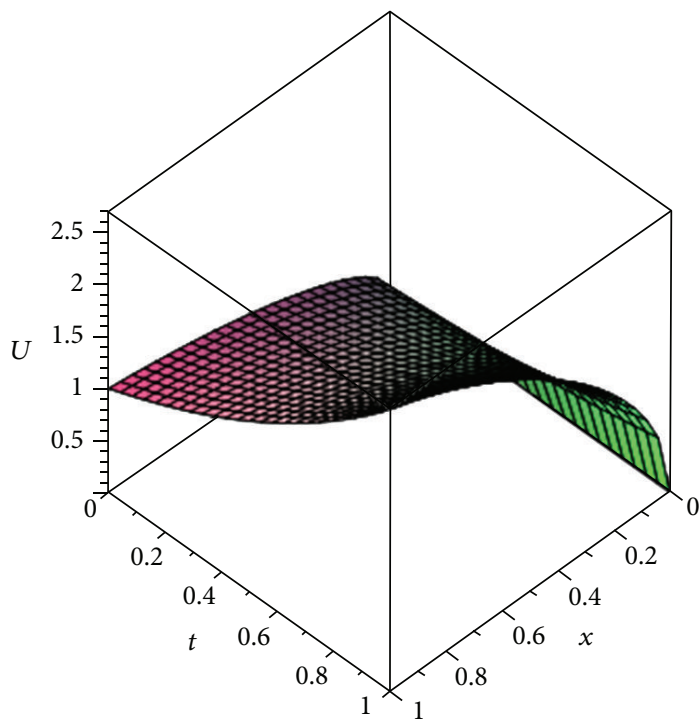

(c)

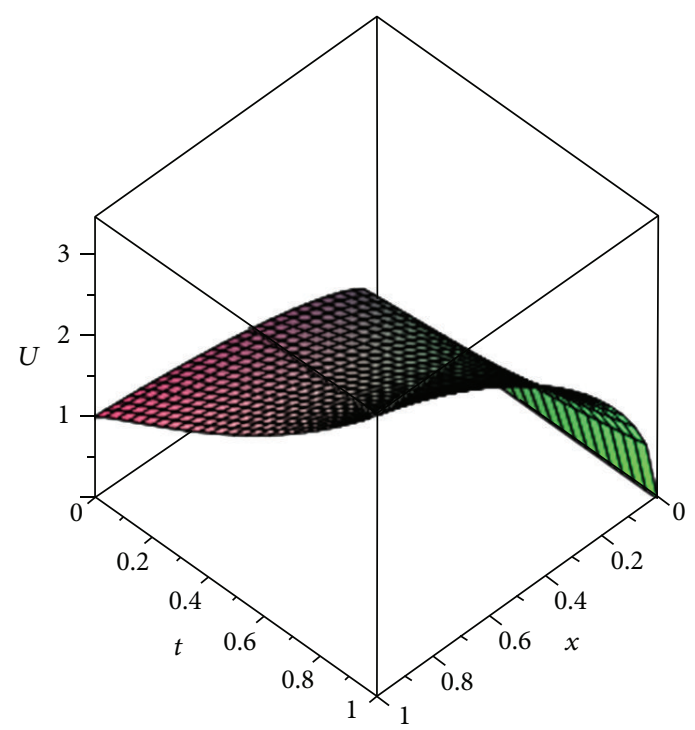

(b)

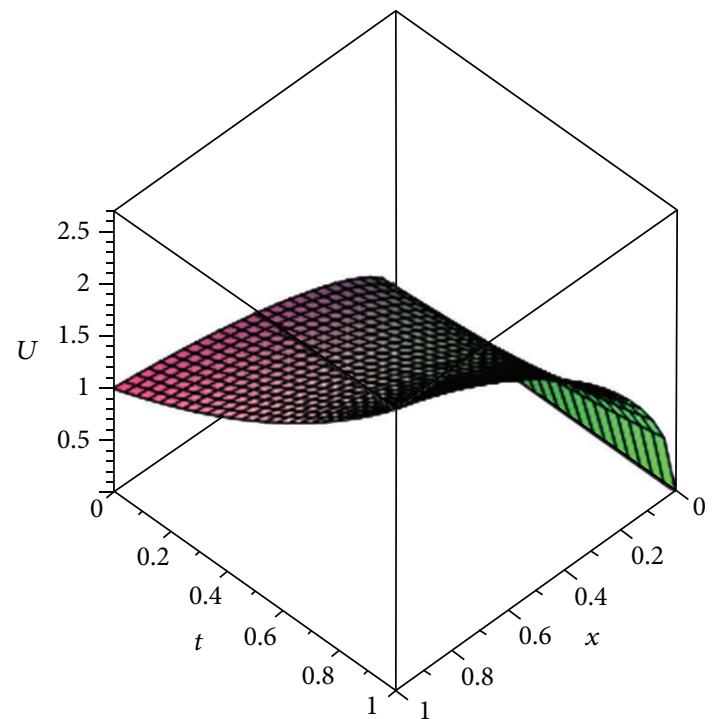

(d)

Figure 4: The behavior of the $U(x, y, t)$ with respect to $x$ and $t$ with $y=1$ is obtained, when (a) $\alpha=0.5$; (b) $\alpha=0.75$; (c) $\alpha=1$; (d) exact solution.

Equating the terms with identical powers of $p$, we have

$$
\begin{aligned}
& p^{0}: U_{0}(x, y, t)=e^{(x+y) / 3}, \\
& p^{1}: U_{1}(x, y, t)=-e^{(x+y) / 3} \frac{t^{\alpha}}{\Gamma(\alpha+1)}, \\
& p^{2}: U_{2}(x, y, t)=e^{(x+y) / 3} \frac{t^{2 \alpha}}{\Gamma(2 \alpha+1)}, \\
& p^{3}: u_{3}(x, y, t)=-e^{(x+y) / 3} \frac{t^{3 \alpha}}{\Gamma(3 \alpha+1)},
\end{aligned}
$$

and so on.
Using (58), the series solution $U(x, y, t)$ is

$$
\begin{gathered}
U(x, y, t)=\lim _{p \rightarrow 1} \sum_{n=0}^{\infty} p^{n} U_{n}(x, y, t) \\
=e^{(x+y) / 3}\left[1-\frac{t^{\alpha}}{\Gamma(\alpha+1)}+\frac{t^{2 \alpha}}{\Gamma(2 \alpha+1)}\right. \\
\left.-\frac{t^{3 \alpha}}{\Gamma(3 \alpha+1)}+\cdots\right] .
\end{gathered}
$$

Taking $\alpha=1$ in (59), we reproduce the solution of the problem as follows: 


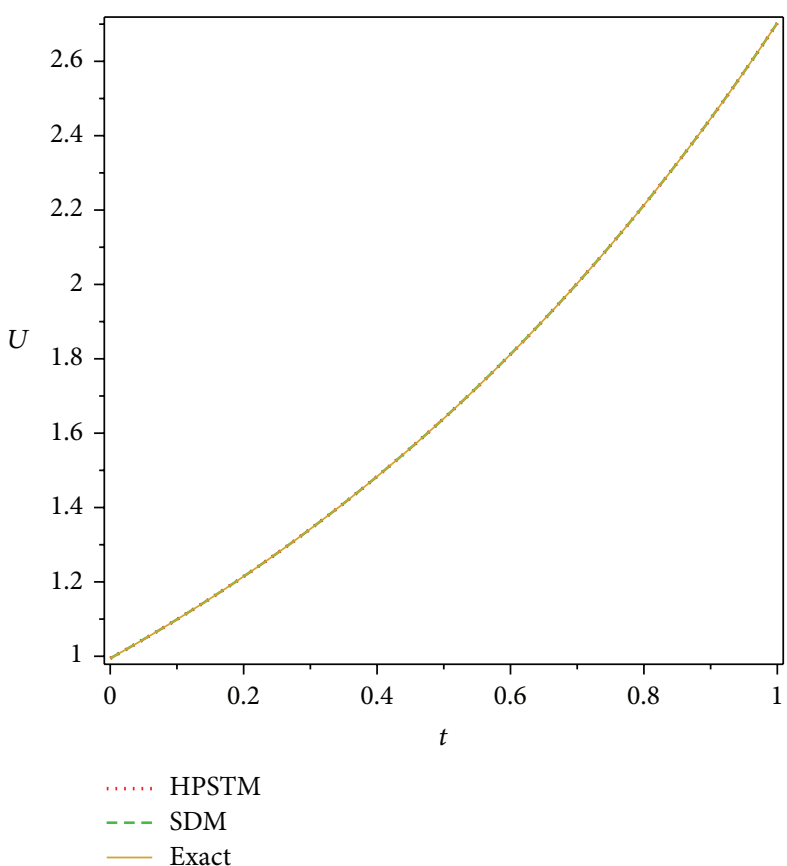

Figure 5: Plots of $U(x, y, t)$ versus $t$ at $x=1, y=1$, and $\alpha=1$.

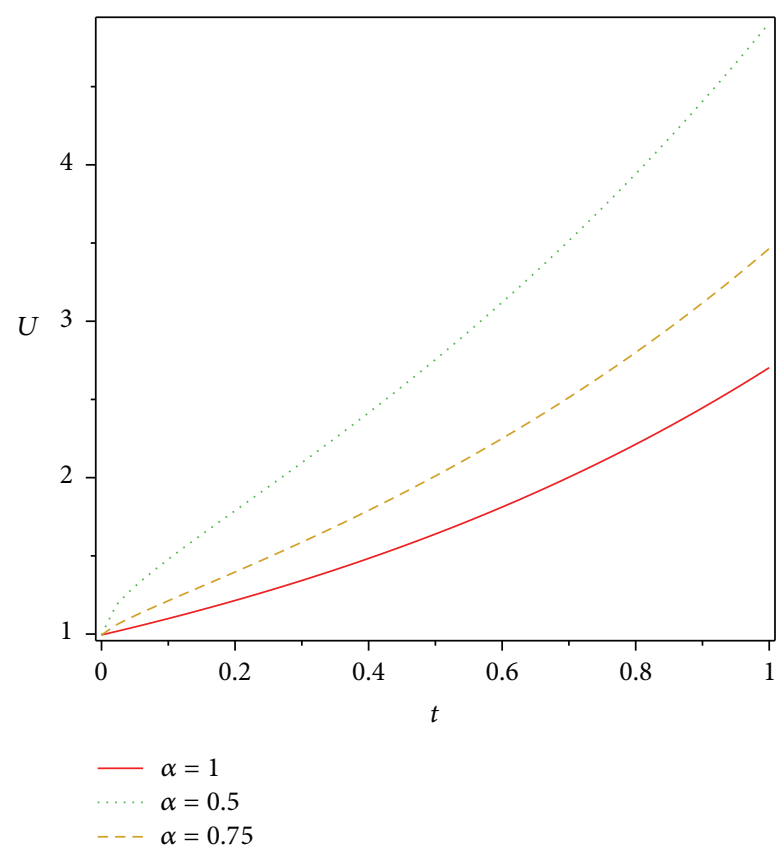

Figure 6: Plots of $U(x, y, t)$ versus $t$ at $x=1$ and $y=1$ for different values of $\alpha$.

$$
U(x, y, t)=e^{(x+y) / 3}\left[1-\frac{t}{1 !}+\frac{t^{2}}{2 !}-\frac{t^{3}}{3 !}+\cdots\right]
$$

which converge very rapidly to the exact solution $U(x, y, t)=$ $e^{(1 / 3)(x+y)-t}$.
According to SDM, we have

$$
\begin{aligned}
\sum_{n=0}^{\infty} U_{n}(x, y, t)= & e^{(x+y) / 3} \\
& +S^{-1}\left(u^{\alpha} S\left[\sum_{n=0}^{\infty} A_{n}-\sum_{n=0}^{\infty} U_{n}(x, y, t)\right]\right) .
\end{aligned}
$$

The recursive relation is given below:

$$
\begin{aligned}
U_{0}(x, y, t) & =e^{(x+y) / 3}, \\
U_{1}(x, y, t) & =S^{-1}\left[u^{\alpha} S\left[A_{0}-U_{0}\right]\right], \\
U_{n+1}(x, y, t) & =S^{-1}\left[u^{\alpha} S\left[A_{n}-U_{n}\right]\right] .
\end{aligned}
$$

The other components of the solution can be easily found by using above recursive relation:

$$
\begin{aligned}
& U_{0}(x, y, t)=e^{(x+y) / 3} \\
& U_{1}(x, y, t)=-e^{(x+y) / 3} \frac{t^{\alpha}}{\Gamma(\alpha+1)}, \\
& U_{2}(x, y, t)=e^{(x+y) / 3} \frac{t^{2 \alpha}}{\Gamma(2 \alpha+1)}, \\
& U_{3}(x, y, t)=-e^{(x+y) / 3} \frac{t^{3 \alpha}}{\Gamma(3 \alpha+1)},
\end{aligned}
$$

and so on.

So, the decomposition series solutions is given by

$$
\begin{aligned}
U(x, y, t)=e^{(x+y) / 3} & {\left[1-\frac{t^{\alpha}}{\Gamma(\alpha+1)}+\frac{t^{2 \alpha}}{\Gamma(2 \alpha+1)}\right.} \\
& \left.-\frac{t^{3 \alpha}}{\Gamma(3 \alpha+1)}+\cdots\right],
\end{aligned}
$$

which is the same solution as obtained by the application of HPSTM and setting $\alpha=1$; it converges to the exact solution $U(x, y, t)=e^{(1 / 3)(x+y)-t}$.

The numerical results for the time-fractional biological population model (55) obtained with the help of HPSTM, SDM, and the exact solution for various values of $t, x$, and $\alpha$ with $y=1$ are described through Figures $7(\mathrm{a})-7(\mathrm{~d})$ and those for various values of $t$ at $x=1, y=1$, and $\alpha=1$ are depicted in Figure 8 and those for different values of $t$ and $\alpha$ at $x=1$ and $y=1$ are presented in Figure 9. It is observed from Figure 7 that $U(x, y, t)$ increases with the increase in $x$ and decreases with increase in $t$ for $\alpha=0.5,0.75$, and 1 with $y=1$. It is to be observed from Figure 9 that as the value of $\alpha$ increase, the value of $U(x, y, t)$ increases, but afterward its nature is opposite. It can also be seen from Figure 7 that the solution obtained by using the present technique is nearly identical with the exact solution. From Figure 8, we can easily observe that the results obtained with help of HPSTM and SDM are in an excellent agreement with the exact solution. It 


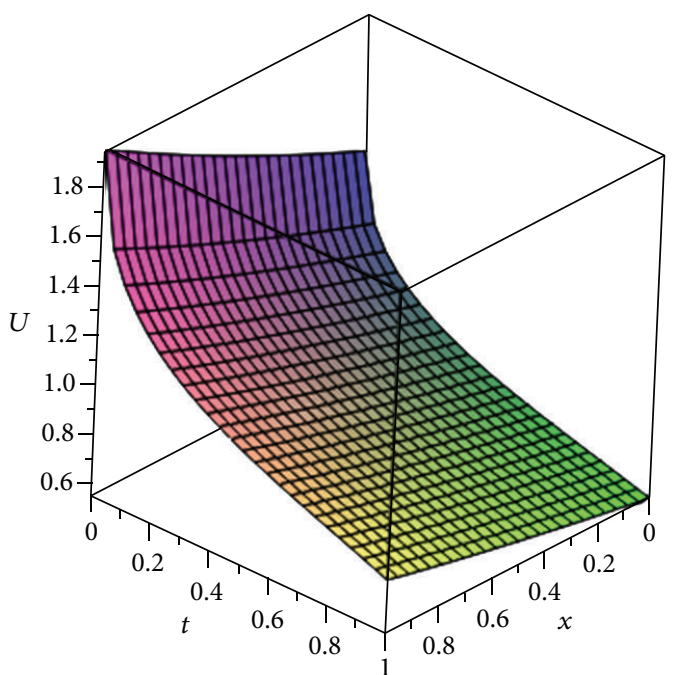

(a)

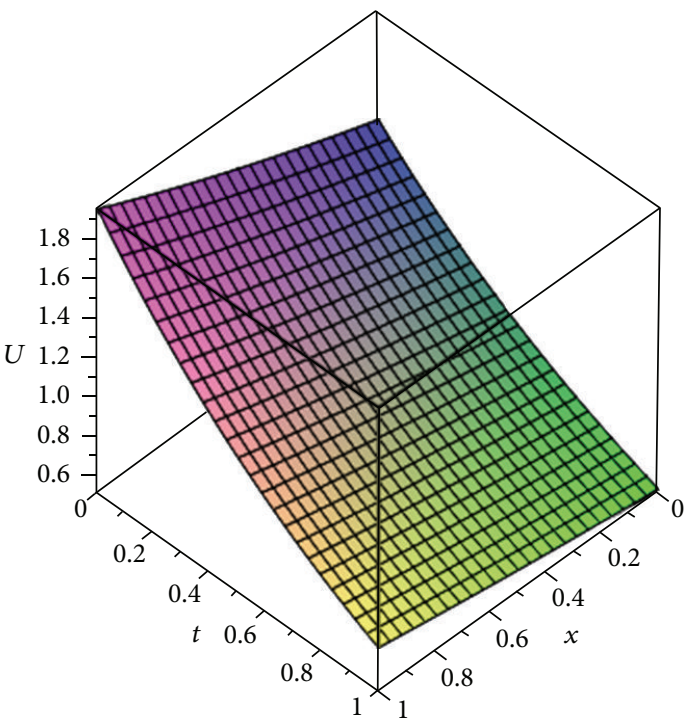

(c)

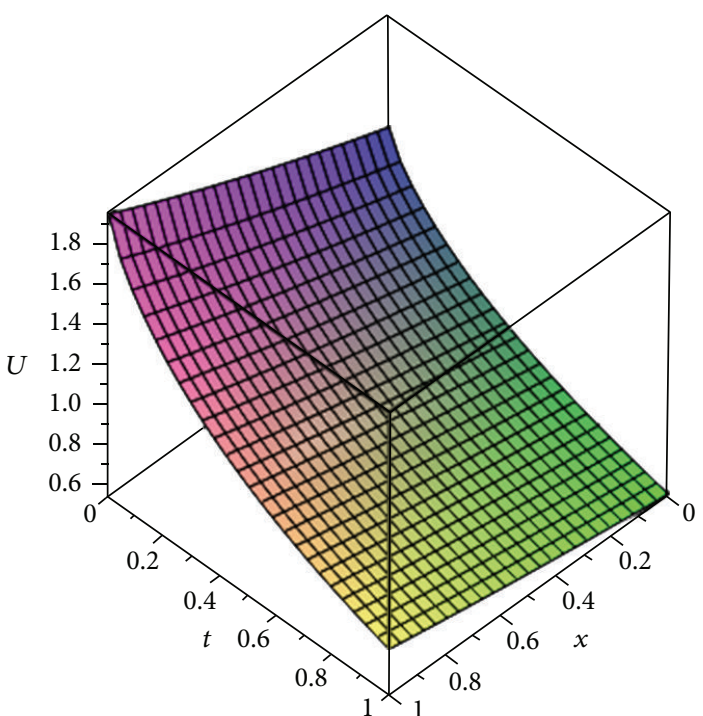

(b)

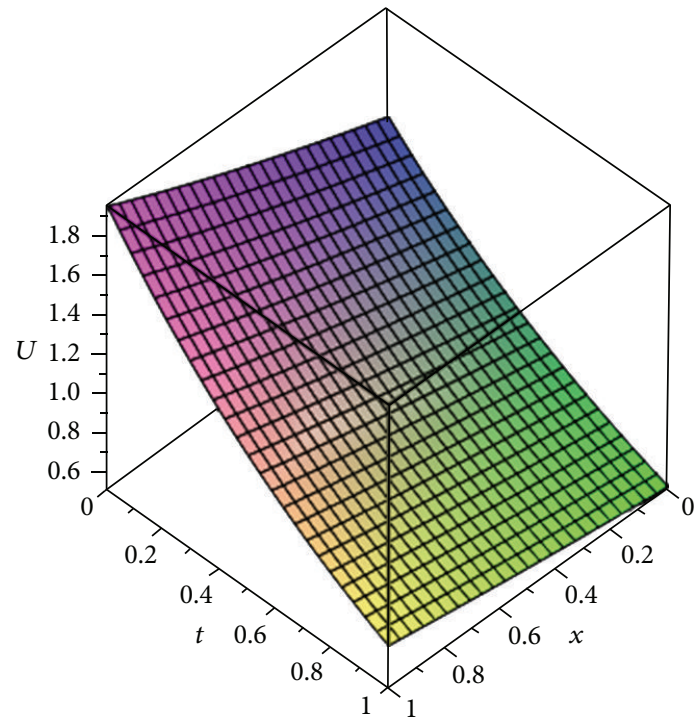

(d)

Figure 7: The behavior of the $U(x, y, t)$ with respect to $x$ and $t$ with $y=1$ is obtained, when (a) $\alpha=0.5$; (b) $\alpha=0.75$; (c) $\alpha=1$; (d) exact solution.

is to be noted that only the seventh order term of the HPSTM and SDM was used in evaluating the approximate solutions for Figures 7 and 8.

\section{Conclusions}

In this paper, the HPSTM and SDM are successfully employed to solve the time-fractional biological population models. The proposed methods provide the solutions in terms of convergent series with easily computable components in a direct way without using linearization, perturbation, or restrictive assumptions. The numerical results obtained with the proposed techniques are in an excellent agreement with the exact solution. It is worth mentioning that both methods are capable of reducing the volume of the computational work as compared to the classical methods while still maintaining the high accuracy of the numerical result; the size reduction amounts to an improvement of the performance of the approach for solving the time-fractional biological population models. Hence, we conclude that the HPSTM and SDM are very powerful and efficient in finding analytical as well as numerical solutions for wide classes of nonlinear fractional partial differential equations arising in science engineering and finance. 


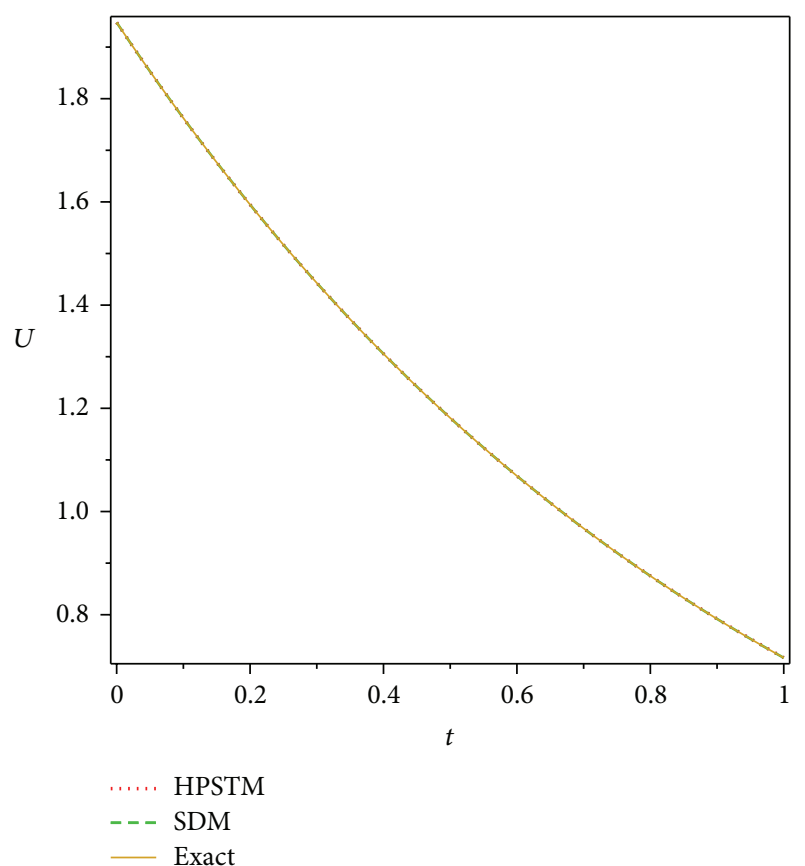

FIgURE 8: Plots of $U(x, y, t)$ versus $t$ at $x=1, y=1$, and $\alpha=1$.

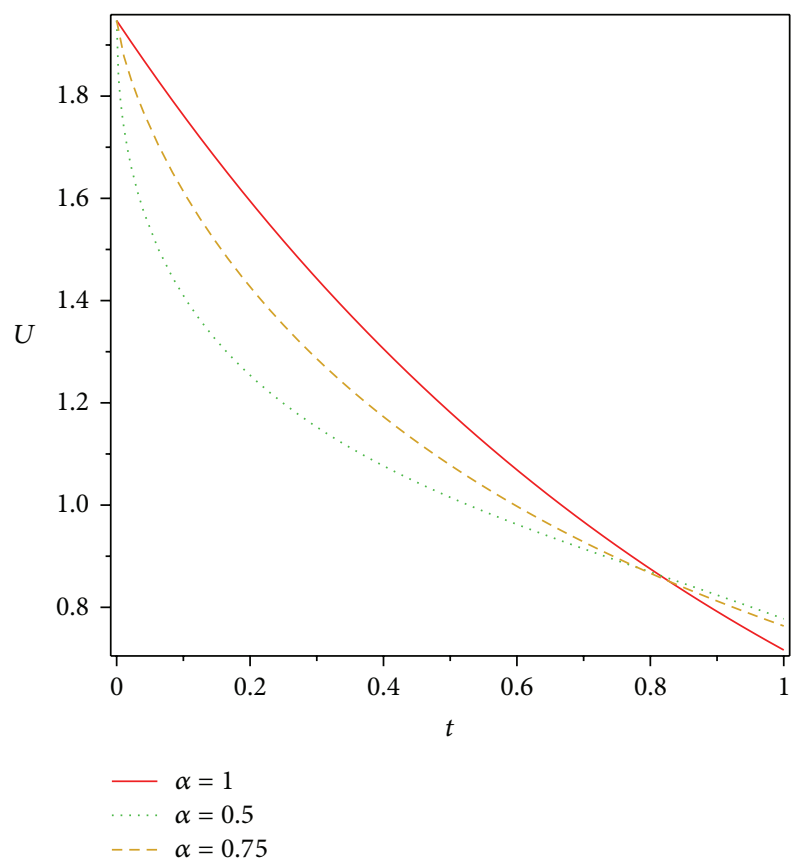

Figure 9: Plots of $U(x, y, t)$ versus $t$ at $x=1$ and $y=1$ for different values of $\alpha$.

\section{Conflict of Interests}

The authors declare that there is no conflict of interests regarding the publication of this paper.

\section{Acknowledgments}

The authors are extending their heartfelt thanks to the reviewers for their valuable suggestions for the improvement of the paper. The authors also gratefully acknowledge that this research was partially supported by the University Putra Malaysia under the GP-IBT Grant Scheme having Project no. GP-IBT/2013/9420100.

\section{References}

[1] J. Bear, Dynamics of Fluids in Porous Media, American Elsevier, New York, NY, USA, 1972.

[2] A. Okubo, Diffusion and Ecological Problem: Mathematical Models, vol. 10 of Biomathematics, Springer, Berlin, Germany, 1980.

[3] M. E. Gurtin and R. C. MacCamy, "On the diffusion of biological populations," Mathematical Biosciences, vol. 33, no. 12, pp. 35-49, 1977.

[4] I. Podlubny, Fractional Differential Equations, Academic Press, New York, NY, USA, 1999.

[5] M. Caputo, Elasticita e Dissipazione, Zani-Chelli, Bologna, Italy, 1969.

[6] R. Gorenflo, F. Mainardi, E. Scalas, and M. Roberto, "Fractional calculus and continuous time finance III: the diffusion limit," in Mathematical Finance, Trends in Mathematics, pp. 171-180, Birkhäuser, Basel, Switzerland, 2001.

[7] R. Hilfer, Ed., Applications of Fractional Calculus in Physics, World Scientific Publishing Company, Singapore, 2000.

[8] K. S. Miller and B. Ross, An Introduction to the Fractional Calculus and Fractional Differential Equations, John Wiley \& Sons, New York, NY, USA, 1993.

[9] K. B. Oldham and J. Spanier, The Fractional Calculus Theory and Applications of Differentiation and Integration to Arbitrary Order, Academic Press, New York, NY, USA, 1974.

[10] A. A. Kilbas, H. M. Srivastava, and J. J. Trujillo, Theory and Applications of Fractional Differential Equations, Elsevier Science, Amsterdam, The Netherlands, 2006.

[11] A. M. A. El-Sayed, S. Z. Rida, and A. A. M. Arafa, "Exact solutions of fractional-order biological population model," Communications in Theoretical Physics, vol. 52, no. 6, pp. 992996, 2009.

[12] A. A. M. Arafa, S. Z. Rida, and H. Mohamed, "Homotopy analysis method for solving biological population model," Communications in Theoretical Physics, vol. 56, no. 5, pp. 797800, 2011.

[13] A. Basiri Parsa, M. M. Rashidi, O. Anwar Bég, and S. M. Sadri, "Semi-computational simulation of magneto-hemodynamic flow in a semi-porous channel using optimal homotopy and differential transform methods," Computers in Biology and Medicine, vol. 43, no. 9, pp. 1142-1153, 2013.

[14] H. N. Hassan and M. M. Rashidi, "Analytical solution for three-dimensional steady flow of condensation film on inclined rotating disk by optimal homotopy analysis method," Walailak Journal of Science and Technology, vol. 10, no. 5, pp. 479-498, 2013.

[15] M. M. Rashidi, E. Momoniat, and M. Ferdows, "Lie group solution for free convective flow of a nanofluid past a chemically reacting horizontal plate in a porous media," Mathematical Problems in Engineering, vol. 2014, Article ID 239082, 21 pages, 2014.

[16] J. H. He, "Homotopy perturbation technique," Computer Methods in Applied Mechanics and Engineering, vol. 178, no. 3-4, pp. 257-262, 1999. 
[17] J. He, "A coupling method of a homotopy technique and a perturbation technique for non-linear problems," International Journal of Non-Linear Mechanics, vol. 35, no. 1, pp. 37-43, 2000.

[18] J.-H. He, "Some asymptotic methods for strongly nonlinear equations," International Journal of Modern Physics B, vol. 20, no. 10, pp. 1141-1199, 2006.

[19] D. D. Ganji, “The application of He's homotopy perturbation method to nonlinear equations arising in heat transfer," Physics Letters A, vol. 355, no. 4-5, pp. 337-341, 2006.

[20] H. Jafari, M. Zabihi, and M. Saidy, "Application of homotopyperturbation method for solving gas dynamics equation," Applied Mathematical Sciences, vol. 2, no. 48, pp. 2393-2396, 2008.

[21] M. M. Rashidi, D. D. Ganji, and S. Dinarvand, "Explicit analytical solutions of the generalized BURger and BURgerFisher equations by homotopy perturbation method," Numerical Methods for Partial Differential Equations, vol. 25, no. 2, pp. 409-417, 2009.

[22] M. M. Rashidi and D. D. Ganji, "Homotopy perturbation combined with Padé approximation for solving two dimensional viscous flow in the extrusion process," International Journal of Nonlinear Science, vol. 7, no. 4, pp. 387-394, 2009.

[23] S. A. Khuri, "A Laplace decomposition algorithm applied to a class of nonlinear differential equations," Journal of Applied Mathematics, vol. 1, no. 4, pp. 141-155, 2001.

[24] M. Khan and M. Hussain, "Application of Laplace decomposition method on semi-infinite domain," Numerical Algorithms, vol. 56, no. 2, pp. 211-218, 2011.

[25] Y. Khan and Q. Wu, "Homotopy perturbation transform method for nonlinear equations using He's polynomials," Computers \& Mathematics with Applications, vol. 61, no. 8, pp. 19631967, 2011.

[26] D. Kumar, J. Singh, and S. Rathore, "Sumudu decomposition method for nonlinear equations," International Mathematical Forum, vol. 7, no. 11, pp. 515-521, 2012.

[27] J. Singh, D. Kumar, and A. Kılıçman, "Homotopy perturbation method for fractional gas dynamics equation using Sumudu transform," Abstract and Applied Analysis, vol. 2013, Article ID 934060, 8 pages, 2013.

[28] G. Adomian, Solving Frontier Problems of Physics: The Decomposition Method, Kluwer Academic Publishers, Boston, Mass, USA, 1994.

[29] G. Adomian and S. E. Serrano, "Stochastic contaminant transport equation in porous media," Applied Mathematics Letters, vol. 11, no. 1, pp. 53-55, 1998.

[30] H. Jafari, S. Ghasempour, and C. M. Khalique, "Comments on "He's homotopy perturbation method for calculating adomian polynomials",' International Journal of Nonlinear Sciences and Numerical Simulation, vol. 14, no. 6, pp. 339-343, 2013.

[31] H. Jafari, S. Ghasempoor, and C. M. Khalique, "A comparison between Adomian's polynomials and He's polynomials for nonlinear functional equations," Mathematical Problems in Engineering, vol. 2013, Article ID 943232, 4 pages, 2013.

[32] G. K. Watugala, "Sumudu transform-a new integral transform to solve differential equations and control engineering problems," International Journal of Mathematical Education in Science and Technology, vol. 24, no. 1, pp. 35-43, 1993.

[33] M. A. Asiru, "Sumudu transform and the solution of integral equations of convolution type," International Journal of Mathematical Education in Science and Technology, vol. 32, no. 6, pp. 906-910, 2001.
[34] F. B. M. Belgacem, A. A. Karaballi, and S. L. Kalla, "Analytical investigations of the Sumudu transform and applications to integral production equations," Mathematical Problems in Engineering, vol. 2003, no. 3, pp. 103-118, 2003.

[35] F. B. M. Belgacem and A. Karaballi, "Sumudu transform fundamental properties investigations and applications," Journal of Applied Mathematics and Stochastic Analysis, vol. 2006, Article ID 91083, 23 pages, 2006.

[36] A. Kılıçman, H. Eltayeb, and M. A. K. Ariffin, "A note on the comparison between Laplace and Sumudu transforms," Bulletin of the Iranian Mathematical Society, vol. 37, no. 1, pp. 131-141, 2011.

[37] A. Kilıcman and H. E. Gadain, "On the applications of Laplace and Sumudu transforms," Journal of the Franklin Institute, vol. 347, no. 5, pp. 848-862, 2010.

[38] V. B. L. Chaurasia and J. Singh, "Application of Sumudu transform in Schrödinger equation occurring in quantum mechanics," Applied Mathematical Sciences, vol. 4, no. 57-60, pp. 2843-2850, 2010. 


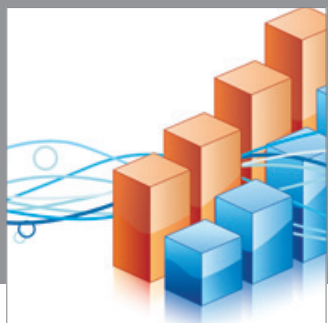

Advances in

Operations Research

mansans

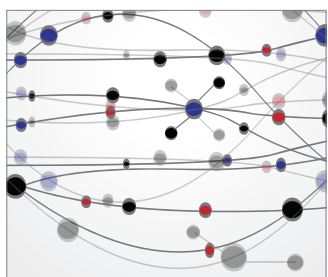

The Scientific World Journal
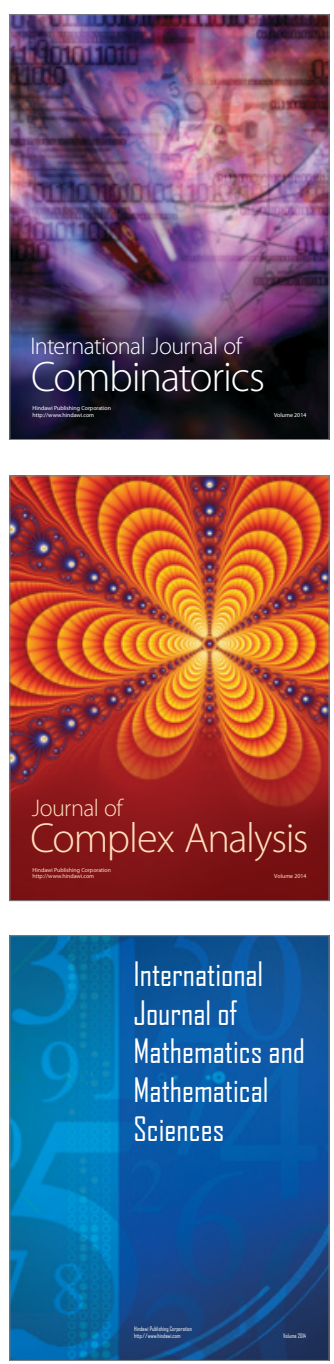
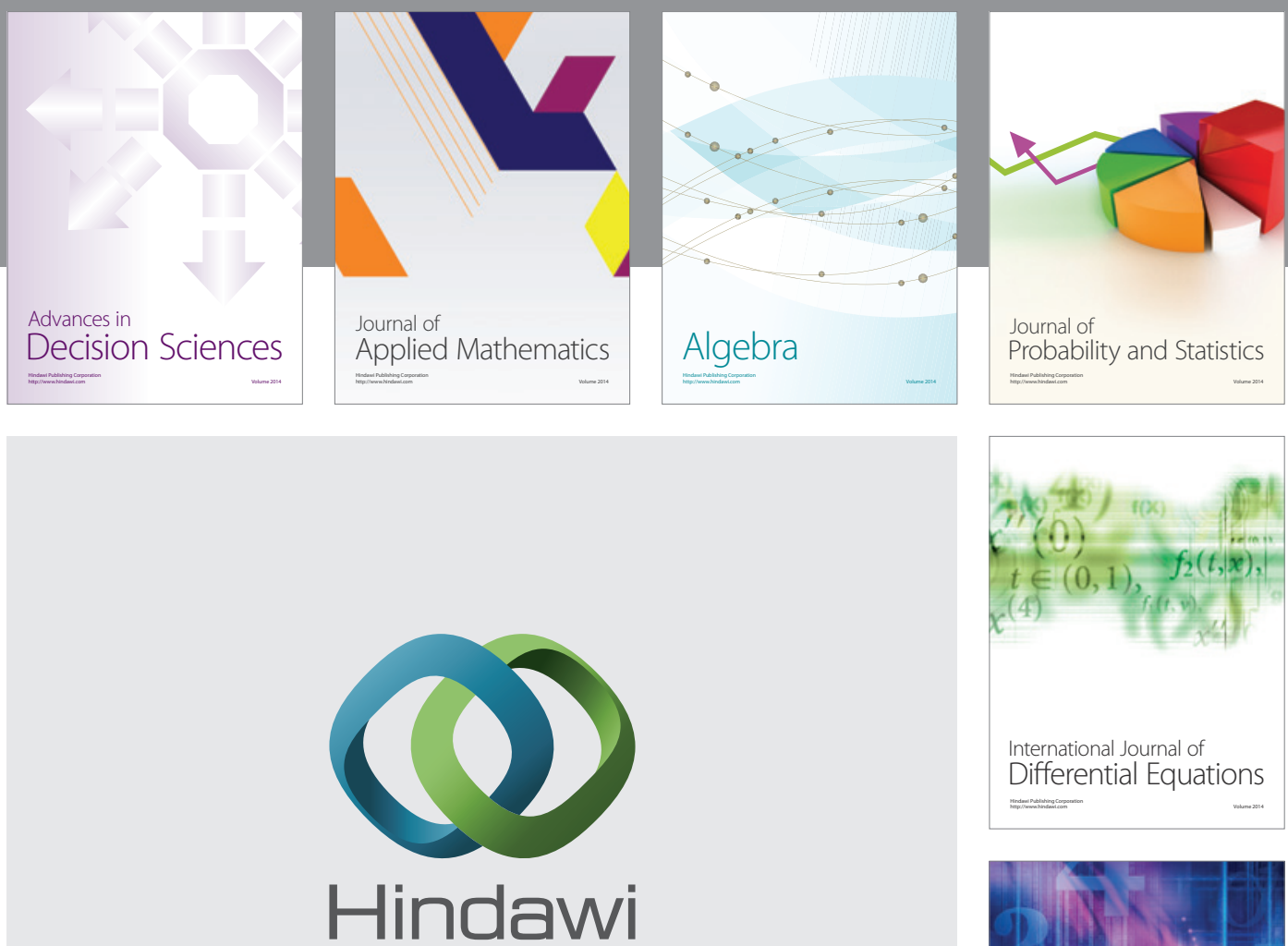

Submit your manuscripts at http://www.hindawi.com
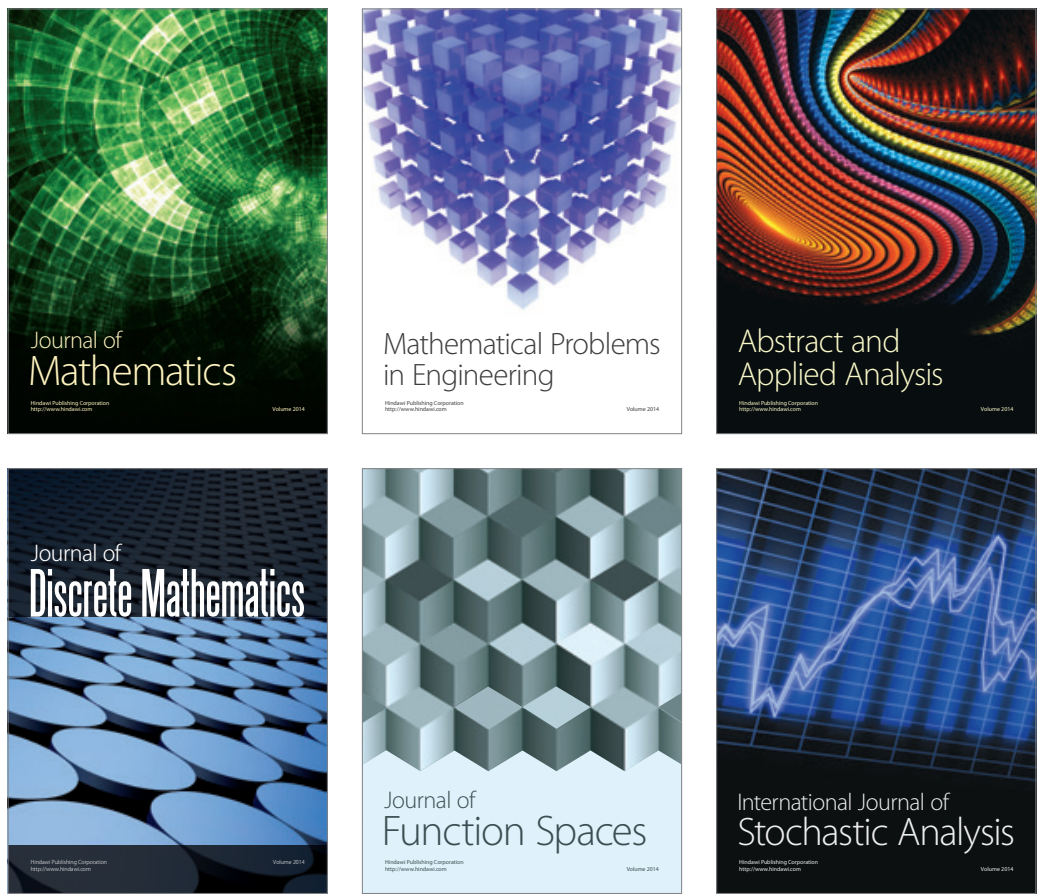

Journal of

Function Spaces

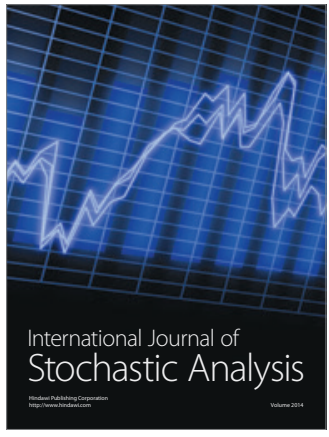

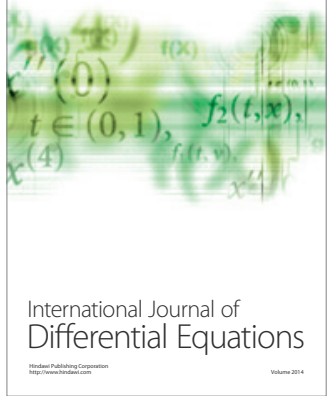
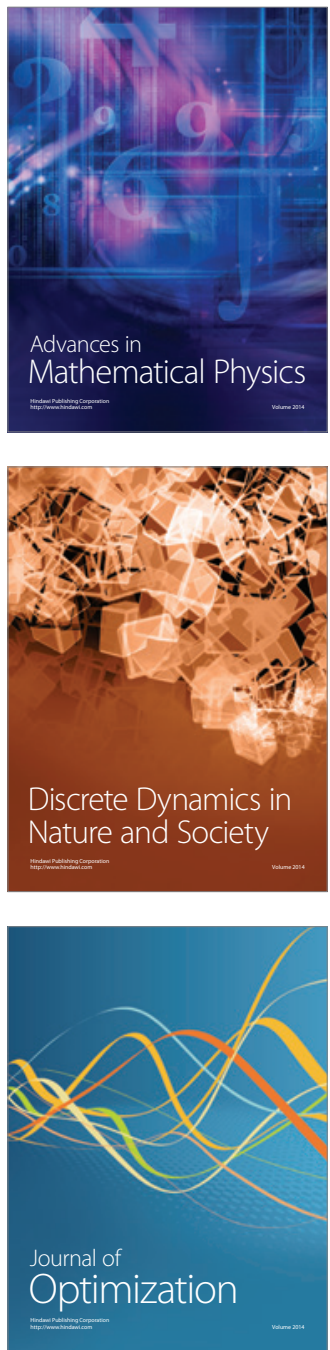